Cahiers « Mondes anciens »

ANCIENS

Histoire et anthropologie des mondes anciens

5 | 2014

Maudire et mal dire : paroles menaçantes en Grèce ancienne

\title{
Injure, honneur et vengeance en Grèce ancienne
}

\section{Manuela Giordano}

\section{(2) OpenEdition}

Journals

Édition électronique

URL : http://journals.openedition.org/mondesanciens/1238

DOI : 10.4000/mondesanciens. 1238

ISSN : 2107-0199

Éditeur

UMR 8210 Anthropologie et Histoire des Mondes Antiques

Référence électronique

Manuela Giordano, «Injure, honneur et vengeance en Grèce ancienne », Cahiers « Mondes anciens » [En ligne], 5 | 2014, mis en ligne le 14 février 2014, consulté le 19 avril 2019. URL : http://

journals.openedition.org/mondesanciens/1238; DOI : 10.4000/mondesanciens.1238

Ce document a été généré automatiquement le 19 avril 2019

\section{(c) $(1)(9)$}

Les Cahiers «Mondes Anciens » sont mis à disposition selon les termes de la licence Creative Commons Attribution - Pas d'Utilisation Commerciale - Pas de Modification 4.0 International. 


\title{
Injure, honneur et vengeance en Grèce ancienne
}

\author{
Manuela Giordano
}

\section{Le statut de l'insulte : une parole dialogique}

$1 \mathrm{Au}$ cours de ce travail je vais éclaircir d'abord - à l'aide de quelques données comparatives - la relation que l'injure entretient avec l'honneur et la vengeance, pour montrer comment injure-honneur-vengeance forment un véritable système. On partira du premier chant de l'Iliade pour arriver à Athènes, pour ébaucher une diachronie de ce système.

2 Il convient au préalable de clarifier le statut de la parole injurieuse. Douée d'une force agressive très marquée, l'injure se situe dans un domaine de réciprocité, d'égalité et d' échange propres au statut de la parole dialogique. Comme je l'ai abordé par ailleurs, en comparant l'insulte, sous la forme littéraire de l'invective (psogos), avec la malédiction ${ }^{1}$, l'injure, en tant qu'arme dans un duel, suppose une syntaxe de rapports paritaires et réciproques, un rival avec lequel on répond du « tac au tac », établissant ainsi un échange. Loin d'être un équivalent de l'injure, la malédiction - tout en étant une parole qui atteint avec violence le destinataire - est une parole n'admettant pas de réplique. Si l'injure est un défi qui exige une réponse, la malédiction, en tant que parole efficace, est plutôt une sentence qui ôte à ses destinataires (ces derniers n'étant pas des « interlocuteurs ») toute possibilité de réponse, proférée d'une position sacrale non-dialogique ${ }^{2}$. Elle agit avec force par la simple action de dire. La puissance de son énonciation peut traverser le temps et l'espace : les erreurs funestes du genos des Atrides ou des Labdacides provoquent des malédictions qui rejailliront sur leurs descendants, parfois à leur insu³.

3 L'injure vit, au contraire, dans l'arène du présent. Elle se nourrit du regard et de la présence physique de l'autre. Elle est un défi qui ébranle cette construction éphémère et vulnérable qu'est l'honneur, dont l'enjeu se mesure exclusivement dans l'espace politique, voire publique. L'injure se comprend tout entière dans une dimension relationnelle et réciproque qui appelle et cause à tout moment la réponse de 
l'interlocuteur, tant elle est entrelacée à la dynamique de l'honneur, de la vengeance, et ainsi à l'identité même de la personne. La nécessité d'une réponse est absolue et vitale si l'on veut garder son honneur. Le risque est de voir sa propre valeur effacée et l'injure transformée en une parole de mépris à l'effet anéantissant ${ }^{4}$.

\section{Insulte et honneur}

4 Depuis les écrits de Peristiany ${ }^{5}$, l'honneur est considéré comme le concept méditerranéen par excellence - ou le concept ayant formé la Méditerranée sur le plan anthropologique, une association qui est depuis longtemps objet de débat ${ }^{6}$. L'honneur est un concept très difficile à saisir et on est loin de s'accorder sur sa définition. Loin de vouloir épuiser ici les questions connexes à l'articulation de ce concept complexe, on rappellera brièvement que l'honneur peut être considéré d'une part comme un code moral, paritaire et égalitaire qui vise à défendre des normes éthiques et le statut d'une famille ou d'un individu, et s'exprime notamment dans la défense des femmes et de leur virginité en tant que dépositaires de la lignée ${ }^{7}$. D'autre part, dans une approche différente mais complémentaire, l'honneur peut se définir comme une idéologie de défense du patrimoine et du groupe familial ${ }^{8}$. Chez les Grecs, je dirais, de façon sommaire et même grossière, que la timè (l'honneur) n'est pas un concept unitaire, mais un système qui articule la valeur personnelle à d'autres éléments, et au don en particulier, connexion qui efface la distinction entre un niveau "matériel » et un niveau "éthique », comme on verra. La célèbre querelle entre Agamemnon et Achille, au premier chant de l'Iliade, se déroule autour de la contestation implicite ou explicite de la timè réciproque des deux rois, provoquée par l'attribution-soustraction du geras de Chryseis-Briséis. Il faut rappeler que la valeur personnelle en Grèce antique, en particulier chez Homère, ne renvoie pas à l'intériorité de l'individu, mais se traduit de deux manières différentes : tout d'abord, par des manifestations matérielles, principalement sous la forme de dons, de regalia et d' insignia qui objectivent socialement la valeur de celui qui les reçoit ${ }^{9}$; ensuite, par un discours sur l'individu, sous la forme du chant épique ou de la vox populi, un «être dit »: " un homme n'est pas un héros, il est dit tel [...] : être dit équivaut à être » ${ }^{10}$. Une atteinte à l'un des objets-timè, tout comme une injure verbale, constitue un outrage à la valeur identitaire et met en danger l'individu : «Si je te cédais en tout, je serais appelé un lâche et un homme de rien (deilos te kai outidanos kaleoimi) " (Iliade, I, 294). Par ces mots, Achille, à la fin de sa querelle avec Agamemnon, motive sa réaction. Le verbe kaleoimi, «je serais dit » renvoie aussi bien à l'« être dit », noyau fondamental constituant la valeur et l'être même de chaque individu, qu'à la passivité qu'implique l'injure restée sans réponse. On voit ainsi s'éclairer davantage le danger de l'injure qui peut aboutir, en dernier ressort, à imposer une nouvelle identité à l'injurié : Achille, le lâche, peut prendre la place d'Achille, le brave. On voit ici la nécessité de répondre du «tac au tac», dans un mécanisme de rétribution et de vengeance qui peut aboutir à l'homicide ${ }^{11}$ :

Tout outrage essuyé amoindrit la timè, lèse la considération sociale de la victime et de son groupe. Celui qui a subi un tort peut démontrer être plus fort et plus vaillant que son offenseur exclusivement par la vengeance ${ }^{12}$.

5 L'honneur-timè mobilise donc un réseau complexe fait d'interactions sociales, d'objets et de discours dont l'injure représente à la fois une partie et, comme on le verra, une mise à l'épreuve. 


\section{L'insulte dans les « sociétés à vengeance» : quelques données comparatives}

6 Entre insulte, honneur et vengeance de sang, il existe évidemment une relation de continuité car l'homicide n'est qu'une forme extrême d'insulte et d'atteinte à l'honneur d'une famille et, par là, à chacun de ses membres. Entre insulte verbale et homicide, dans ce sens, il n'y a qu'une différence de degré, et non de nature. De manière analogue, la vengeance se situe dans le domaine de la réciprocité et, comme on l'a souligné depuis les travaux de Verdier, de l'échange ${ }^{13}$.

7 On partira de quelques données comparatives tirées de deux " sociétés à vengeance » ( feuding societies) qu'on peut considérer comme un cas particulier des «sociétés à honneur ». Il s'agit de sociétés qui entrent de plein droit dans la catégorie des sociétés de «face-à-face » et dans lesquelles les paroles, plus qu'ailleurs, «font des choses $»^{14}$. Dans ces sociétés, l'injure verbale est l'une des actions qui menacent et blessent l'intégrité d'un individu ou d'un groupe familial. Une telle injure nécessite une réponse qui rétablit l'équilibre de l'honneur et restitue l'intégrité de la personne ou du groupe familial par une action de type rétributif, à savoir une réponse vindicatoire pouvant inclure l'homicide, c'est-à-dire la vengeance de sang. Parmi les nombreux exemples, nous ne retiendrons que deux cas bien étudiés qui présentent des analogies intéressantes avec le cas grec antique ${ }^{15}$.

8 Chez les Sarakatsani, un groupe de bergers transhumants des montagnes de Zagori (4000 personnes), étudié dans les années 1960 par Campbell, l'honneur [= timé (!)] régit entièrement la vie sociale du groupe et les comportements individuels. Cette timé est bien comparable à la timè homérique en tant que valeur que la communauté confère à un individu en reconnaissant son excellence ${ }^{16}$. Une des menaces les plus sévères pour la timé est justement l'insulte verbale, comparée à la violence physique, l'homicide, la séduction ou le viol d'une femme du groupe familial ${ }^{17}$. La classe d'âge dont la timé est maximale est celle des hommes non mariés ${ }^{18}$. Chez eux, cette obsession de l'honneur se traduit par une grande sensibilité aux insultes :

It is not only the reality of an obvious insult which provokes him to action, but even the finest of allusions on which it is possible to place some unflattering construction ${ }^{19}$.

Dans cette remarque de Campbell, on décèle toute la fragilité de la construction sur laquelle repose cet honneur et le fait que l'honneur et le statut social de chacun sont constamment mis à l'épreuve. La réponse vindicative est toutefois souvent tempérée par la médiation des autres hommes du groupe et les homicides d'honneur restent relativement rares.

Honneur et vengeance occupent aussi une place absolument centrale dans la culture albanaise traditionnelle qui s'exprime notamment dans son droit oral, le Kanun, un ensemble très ancien de normes coutumières, rédigé sous forme écrite au début du XIx siècle, dans le Kanuni $i$ Lek Dukagjinit (Kanun de Lek Dukagini) ${ }^{20}$. Il correspond au code traditionnel des montagnards du Nord ${ }^{21}$. Le cas albanais est fort intéressant car, dans ce pays, la pratique de la vengeance pour défendre l'honneur est très répandue et considérée comme une vraie plaie sociale - au point d'ailleurs que le risque de vengeance peut légitimer la demande d'obtention d'un permis de séjour pour raisons humanitaires ${ }^{22}$. 
11 Le Kanun édicte que l'honneur, nderi, « est un patrimoine personnel, et personne ne peut empêcher, avec des moyens judiciaires, le rachat de l'honneur. L'honneur a été imprimé sur le front (scil. de l'Albanais) par Dieu souverain $»^{23}$. Le Kanun inventorie encore des cas précis où l'homme doit se considérer comme déshonoré (§ 593-639). On dira toutefois, avec Resta, qu'en Albanie «l'honneur est un langage, un récipient sémantique vide, et donc ductile, doté de frontières flottantes, et sujet à interprétation, mais lié à ce que, dans leur vie, les Albanais apprennent à considérer comme des faits réels, un code qui imprime un sens à la réalité ${ }^{24}$. Dans cette rhétorique de l'honneur, la réponse par la vengeance à l'injure et à toute forme d'outrage revêt un caractère automatique et nécessaire, le risque étant d'être considéré comme déshonoré, ce qui équivaut à perdre son identité ; le Kanun, au paragraphe 600, dit clairement que : « devant la loi le déshonoré est considéré comme une personne morte $»^{25}$.

12 Resta remarque que « l'injure, en connexion avec la violation d'un patrimoine, suppose l'atteinte au prestige d'un homme et trahit son incapacité à protéger les membres du groupe, entame sa réputation et demande vengeance $»^{26}$, et encore que «toute injure est perçue comme un affront à venger et présuppose des notions de prestige, d'honneur et de virilité qui ramènent au fait d'être un homme $\aleph^{27}$. Martucci ${ }^{28}$ relate un entretien datant de juillet 2009 avec Hil Vila, un kryeplak, un chef de village de Rranxa près de Skodr, dans le nord du pays témoin d'un homicide d'honneur survenu en 1995. Lui-même a été par la suite impliqué dans une chaîne de vengeances, et il décrit ainsi le début de la querelle :

En novembre 1995 je me trouvais dans un restaurant pour dîner. Il y avait beaucoup de monde dans ce restaurant, il y avait aussi un monsieur d'un village proche d'ici qu'on connaissait bien, nos parents étaient amis. On ne sait pas bien ce qui s'est passé, s'ils ont bu un peu trop ou quoi d'autre... moi, j'étais là ce soir-là par hasard. Nous étions loin et, à un moment donné, ils ont commencé à se disputer. Je me suis levé parce que je connaissais cet Anton et que, comme j'étais plus vieux, j'étais en position de calmer la situation. Mais il était très nerveux, et il a commencé à lancer des injures. Les insultes, d'après nos règles, d'après le Kanun, sont en soi très graves, et, après l'avoir entendu proférer des insultes de façon répétée, un homme de ma famille, armé lui aussi, lui a tiré dessus et l'a tué, sans que personne ne s'en aperçoive.

Dans ce récit, on voit se déployer un modèle typique : ivresse, dispute dans un lieu public, insultes, homicide ${ }^{29}$. Dans tous ces cas l'insulte, parfois adressée à une femme du groupe familial, est perçue comme une atteinte à l'honneur d'un homme (et de la famille) et constitue un vulnus à son intégrité, qui doit être vengée.

\section{Un échange d'injures : Achille et Agamemnon}

14 Parmi les nombreux exemples homériques de réponses vindicatoires à l'atteinte à l'honneur, la fameuse querelle entre Achille et Agamemnon, qui occupe le cœur du premier chant de l'Iliade, est le cas le plus riche et le plus articulé. Je ne m'attarderai pas ici sur les aspects politiques et institutionnels qui ont déjà fait l'objet d'autres études et interventions $^{30}$; je chercherai plutôt à amorcer le parcours des mots et leur transformation à la lumière des points énoncés plus haut ${ }^{31}$.

La querelle se déroule au cours de l'assemblée guerrière, occasion publique et agonale par excellence, pendant laquelle les chefs et les simples soldats se trouvent littéralement face à face et mesurent, chacun en fonction de son rôle, leur honneur ${ }^{32}$. La querelle débute par la contestation par Achille de la décision du roi Agamemnon, à savoir qu'il doit recevoir 
un autre geras pour remplacer celui constitué par la prisonnière Chryseis qui lui a été assignée, mais qu'il doit restituer à son père :

Illustre fils d'Atrée, pour la cupidité, tu n'as pas ton pareil ! Et comment les Achéens magnanimes pourraient-ils te donner semblable part d'honneur? Nous n'avons pas, que je sache, de trésor commun en réserve. Tout ce que nous avons tiré du sac des villes a été partagé: il ne serait pas coutumier que les gens de nouveau le rapportent à la masse ${ }^{33}$.

L'intervention d'Achille se présente comme un rappel aux règles communautaires et une exhortation faite à Agamemnon afin qu'il revienne sur sa décision. Or, pour le roi Atride, comme on le verra dans sa réponse, il s'agit d'un défi. En termes d'honneur, il revient à la timè d'Agamemnon en tant que roi " plus roi » et plus puissant que les autres de décider. Contester sa décision signifie contester son honneur et son rôle parmi les Achéens. On trouvera à ce propos utile de citer la définition de Stewart sur l'honneur réflexif, reflexive honor: «if A impugns B's honor, then B's honor is ipso facto diminished or destroyed, unless $B$ responds with an appropriate counterattack on $A »^{34}$. Agamemnon (B) est contesté par Achille (A) et son autorité se trouve ipso facto amoindrie : s'il ne répond pas, il risque de voir contester son image sociale et son rôle, en somme sa timè de chef, face aux autres Achéens. Mais Agamemnon ne recule pas et entre dans le domaine agonistique en débutant ainsi la vraie querelle :

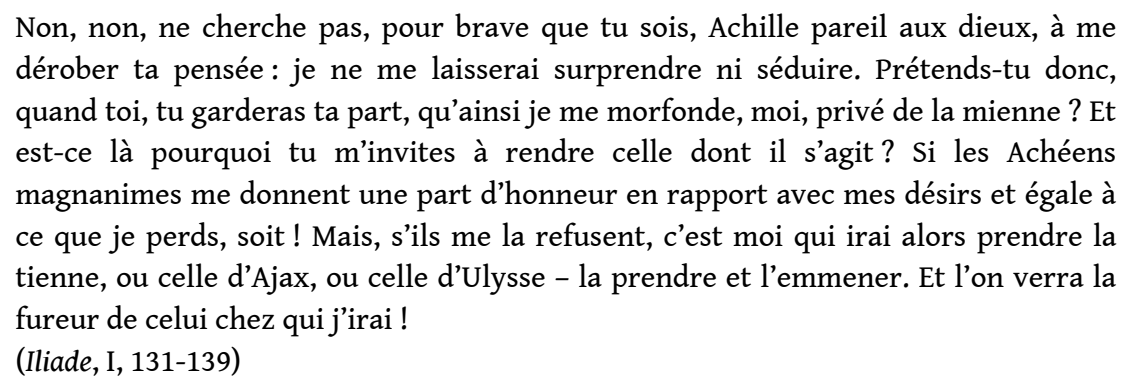

17 La menace de soustraire le geras d'un autre chef est à son tour une atteinte à la timè d'Achille qui répond à Agamemnon par des insultes (anaideièn epiemene, kerdaleophron, v. 149 ; anaides, v. 158, kynôpa, v. 159) et par une véritable déconstruction de son autorité de basileus :

Ah! Cœur vêtu d'effronterie et qui ne sait songer qu'au gain! Comment veux-tu qu'un Achéen puisse obéir de bon cœur à tes ordres, qu'il doive aller en mission ou marcher à un franc combat ? C'est toi, toi, l'effronté que nous avons suivi, pour te plaire, pour vous obtenir aux frais des Troyens une récompense, à vous, Ménélas et toi, face de chien ! (v. 153-157).

18 Dans les mots d'Achille, Agamemnon ne dispose pas de toutes les qualités requises pour un «leadership » royal pouvant jouir d'autorité : bravoure, courage, adresse militaire, charisme, bon conseil et connaissance des règles communautaires et du système des timai ${ }^{35}$. On rappellera qu'être traité de couard est l'une des insultes les plus graves, non seulement chez Homère, mais aussi dans l'Athènes classique, où un déserteur pouvait être frappé d'atimie ${ }^{36}$. De plus, Agamemnon n'est pas capable de susciter l'obéissance guerrière chez les Achéens, au point que le fils de Pelée menace de partir plutôt que d'être déshonoré (atimos, v. 171). Une menace qu'il réalisera bientôt en renonçant à combattre. La réponse d'Agamemnon méprise la valeur d'Achille en l'invitant à partir et termine par l'outrage final :

Si Phoibos Apollon m'enlève Chryséis, je la ferai mener par une nef et des hommes à moi ; mais, à mon tour, en personne, j'irai jusqu'à ta baraque, et j'en emmènerai la jolie Briséis, ta part, à toi, pour que tu saches combien je suis plus fort que toi, et 
que tout autre à l'avenir hésite à me parler comme on parle à un pair et à s'égaler à moi devant moi. Sarakatsani, très attaché à son honneur et prompt à répondre à l'insulte par tous les moyens, y compris le meurtre, Achille, jeune homme de valeur indiscutée, songe immédiatement à tuer Agamemnon, tout en considérant l'alternative, se contrôler (v. 188-192). Il tire déjà l'épée du fourreau quand Athéna - véritable hypostase de la maitrise de soi - intervient pour retenir la fureur du fils de Pélée (v. 193 sq.), le tire par les cheveux et le raisonne :

Allons! clos ce débat, et que ta main ne tire pas l'épée. Contente-toi de mots, et, pour l'humilier, dis-lui ce qui l'attend. Va, je te le déclare, et c'est là ce qui sera : on t'offrira un jour trois fois autant de splendides présents pour prix de cet outrage. Contiens-toi et obéis-nous.

21 Le déplacement proposé par Athéna, de l'agressivité physique à l'agressivité verbale, est tout à fait significatif: le meurtre est remplacé par une attaque verbale d'insultes ( oneidison epesin) qui transfère l'agression de l'épée aux mots. Le transfert d'agressivité est bien étudié par les anthropologues dans le contexte guerrier, où l'injure sert à la ritualisation du conflit. Rappaport décrit ainsi les guerres hautement ritualisées des Tsembaga de la Nouvelle Guinée ${ }^{37}$. La bataille est précédée d'une phase très élaborée (qui vise en fait à éviter le déroulement de la bataille elle-même) pendant laquelle, après d'autres procédures, les adversaires se rapprochent les uns des autres, à une distance telle qu'ils peuvent s'entendre : ils commencent alors à se lancer des injures qui ont pour fonction d'exprimer leur rage et d'essayer de la canaliser. Cette phase peut d'ailleurs conduire à une conciliation. Dans une perspective éthologique, Eibl-Eibesfeldt mentionne encore les échanges d'injures comme forme de ritualisation du conflit dans le cadre général du contrôle culturel de l'agressivité, qui vise justement à éviter les assassinats potentiels. Les exemples sont nombreux et vont des duels des Esquimaux, qui se manifestent exclusivement par des chants sarcastiques, aux duels verbaux des Tyroliens ou des Afro-américains qui remplissent tous, de diverses façons, la fonction d'exutoire qui, du point de vue phylogénétique, joue le rôle fondamental pour l'espèce humaine de contrôler l'agressivitée ${ }^{38}$.

Ainsi Achille, suivant le conseil d'Athéna, contrôle cette agressivité qui l'aurait amené à tuer Agamemnon. Il canalise sa rage verbalement et atteint de flèches mortelles l'image d'Agamemnon, en le traitant à nouveau de lâche, "œil de chien et cœur de cerf », chef militaire raté et avide : il est un roi, oui, mais un dèmoboros basileus, un roi qui dévore son peuple ${ }^{39}$.

En conclusion, la querelle déploie de façon paradigmatique les dynamiques mobilisées par la triade injure-honneur-vengeance. L'enjeu de la querelle est l'honneur dans le sens de valeur individuelle et d'excellence. En rappelant la définition d'honneur chez les Sarakatsani comme reconnaissance d'excellence d'un individu par la communauté, on voit clairement que la querelle entre Achille et Agamemnon est un défi d'excellence : d'une part, dans le domaine de l'autorité et du pouvoir d'Agamemnon et, d'autre part, dans le domaine de la suprématie guerrière d'Achille. Mais les domaines s'entrelacent vite et 
l'enjeu devient l'excellence tout court : qui est le meilleur des Achéens, aristos Achaion (cf. Iliade, I, 94 et 244)? Il est utile ici de rappeler la définition donnée par Donlan du « système fluide » qui caractérise le monde homérique. Dans ce système, la timè est ce qui hiérarchise les hommes entre eux et, loin d'être fixée une fois pour toute, celle-ci se gagne ou se perd dans un contexte agonal, lorsque les guerriers homériques se mesurent à l'extérieur du groupe, via la guerre, et à l'intérieur, via un ensemble de conflits ritualisés - analogues aux maints rituels enregistrés par les anthropologues - parmi lesquels notamment les discours en assemblée et les compétitions athlétiques ${ }^{40}$. Il est clair que le type de suprématie gagnée dans ces contextes apparait dynamique, "fluide » et non fixé une fois pour toutes. Il s'agit d'une supériorité qui est, dans les mots de Benveniste «temporaire, toujours remise en jeu $»^{41}$. L'honneur tour à tour disputé par le moyen de l'injure est donc toujours un honneur « horizontal » ${ }^{42}$, c'est-à-dire un honneur entre des pairs ayant même droit de parole et d'insulte ${ }^{43}$. Un homme de rang inférieur ne s'engage pas dans cette épreuve d'honneur, parce qu'il n'a pas assez de force sociale ou physique pour défendre son honneur ${ }^{44}$.

De ces considérations, ressort de manière très claire la valeur agonale de l'injure, parole se jouant dans une arène publique. On peut aller plus loin et dire avec Maravall que « l'estime et le prestige n'apparaîtraient point comme un honneur, si l'outrage n'était pas possible contre cet honneur. Il présuppose donc la succession d'une double attitude, d'attaque et de défense $»^{45}$. L'honneur relie le mécanisme de l'échange d'injures à l'échange meurtre-vengeance, car «le meurtre est ce qui permet, positivement, au groupe de la victime de se définir comme tel». La réponse par la vengeance est l'« occasion la plus spectaculaire, la plus dramatique et sans doute la plus efficace pour le groupe [...] de se mobiliser dans son unité $»^{46}$. En tant que pratique identitaire, la réponse vindicatoire à l'injure amène au résultat constructif et fonctionnel de renforcer l'identité d'un groupe ou d'un individu dans la société.

On peut donc conclure que si l'honneur est agonal en soi et contient dans sa structure le conflit, l'injure a pour fonction de mettre à l'épreuve l'honneur et les règles sociales qui lui sont liées; elle fonctionne en même temps comme une mise à l'épreuve permanente de la valeur et de l'identité. Dès lors, il faut considérer l'injure comme un élément et une pratique intégrés au monde homérique et non pas comme une instance isolée.

\section{La parole armée : comment combattre avec des mots}

Pour conclure cette analyse, on doit revenir sur le statut de la parole injurieuse, en la nourrissant des conclusions précédemment dégagées. Située dans le domaine d'échange publique, la parole d'injure jouit du pouvoir d'altérer l'identité et l'honneur du héros, jusqu'à le meurtrir. Un pouvoir qui va de pair avec celui de la parole publique de louange, ce pouvoir de la parole de "faire l'identité » et de "faire l'honneur», tout comme d'octroyer la reconnaissance de l'excellence. Comme on l'a démontré, ce pouvoir performatif de l'injure est non seulement une partie constitutive des capacités abstraitement verbales d'un guerrier, mais il forme aussi un outillage au potentiel belliqueux. La connotation agressive au sens éthologique de l'échange d'injures entre Achille et Agamemnon, comme véritables armes qui blessent, est bien exprimée par le lexique. La relation des combattants est désignée par le sémantème -ơvv qui définit l'opposition spatiale et proxémique, la position frontale du défi entre les deux adversaires 
qui échangent des insultes ${ }^{47}$. À la fin de la querelle, au moment où l'assemblée est en train de se disperser, le couple antagoniste Achille-Agamemnon est ainsi libéré, aux v. 304-305 :

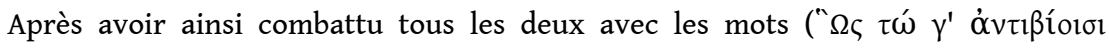

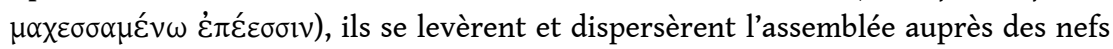
des Achéens.

Les deux rois sont désignés par la forme grammaticale du duel, tout à fait significative

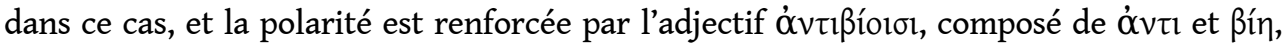
"opposant la force à la force" par des mots qui se combattent. Voici résumée la quintessence de la structure diadique de l'injure : l'un en face de l'autre, les yeux dans les yeux, se combattant avec des mots qui opposent la force à la force ${ }^{48}$. Que l'échange d'insultes relève d'un contexte non seulement agonal au sens générique mais aussi guerrier est démontré par le fait que l'adjectif ơvtíßıoৎ et l'adverbe ờvıßínv sont utilisés dans toutes les autres occurrences attestées dans un contexte militaire, lorsque la force est celle des armes et non des paroles, comme c'est le cas ici.

À la lumière de ces considérations lexicales, des données anthropologiques et éthologiques mentionnées plus haut sur la fonction des injures dans les duels et les combats ritualisés par le moyen de la "parole armée ", on pourra lire à nouveau le célèbre passage qui définit le guerrier homérique comme «un bon parleur aussi bien qu'un acteur d'entreprises». Phénix y rappelle sa fonction d'instructeur auprès d'un jeune Achille, guerrier inexpérimenté (Iliade, IX, 440-443) :

Tu n'étais qu'un enfant, et tu ne savais rien encore ni du combat qui n'épargne personne ni des Conseils où se font remarquer les hommes.

Et c'est pour tout cela qu'il m'avait dépêché : je devais t'apprendre à être en même temps un bon diseur d'avis, un bon faiseur d'exploits.

Le guerrier homérique doit savoir manier les outils d'une rhétorique de bataille, non seulement pour faire valoir sa parole à l'assemblée et au conseil, convaincre les autres et vaincre les adversaires, mais il lui faut aussi une parole qui sache blesser sans dommage son pair dans l'arène interne des égaux. Il doit savoir mettre à l'épreuve l'honneur des autres, tout en évitant le danger d'une violence physique qui aurait des effets terribles de stasis sur la communauté guerrière ${ }^{49}$. Il doit savoir déverser sa rage en la canalisant par des mots armés, et ce sans toucher ses armes.

\section{Conclusions en guise d'abordage au Pirée}

Pour finir, c'est à Athènes qu'il convient de se rendre afin de prolonger la réflexion sur cette rhétorique efficace visant à défendre l'honneur. Être un «bon parleur de mots » était une qualité hautement requise dans une polis où le citoyen moyen devait souvent défendre sa vie, sa famille, ses biens et sa réputation par des procès durant lesquels chacun plaidait sa propre cause - accusation ou défense - avec une urgence pareille à celle ressentie en cas de guerre.

Just as the warriors feel the need to avert and avenge attacks on themselves and their families, so too speakers in the Attic courts refer to the necessity for them to avenge through the courts wrongs done to themselves and their families ${ }^{50}$.

31 En particulier, la possibilité de poursuivre pénalement une insulte (kakègoria) par la dikè kakègorias (et même par la graphè hybreôs) peut être vue comme une partie de la stratégie athénienne pour canaliser la violence dans une voie judiciaire, qui s'inscrit dans le cadre plus vaste d'une régulation des réponses par la vengeance. Je ne dis rien de nouveau en 
affirmant que, chez les Athéniens, se développent des moyens de résolution du conflit en adéquation avec les mécanismes démocratiques, c'est-à-dire plus protecteurs et avantageux pour la communauté, dans une stratégie idéologique où la réponse vindicatoire est jugée obsolète et socialement nuisible et où le traitement de l'insulte revêt une grande importance ${ }^{51}$. Mais l'honneur défendu à Athènes reste un honneur horizontal, comme chez Homère, ainsi que l'a bien exprimé Évelyne Scheid-Tissinier : « la colère suppose, pour pouvoir être déclenchée, l'existence d'une certaine égalité socialement reconnue entre l'agresseur et l'agressé $\aleph^{52}$. Les actions de représailles continuent à postuler une parité de timè entre l'offenseur et l'offensé ${ }^{53}$. La stratégie civique athénienne intégre l'ancien dans le nouveau, avec des mises à jour et des sémantisations nouvelles ${ }^{54}$. La partie la plus importante de cette stratégie est une opération rhétorique du discours public autour du modèle du contrôle de soi, la sôphrosynè : le citoyen athénien est celui qui est capable de contrôler ses impulsions et de ne pas céder à la colère, dans la phalange hoplitique comme dans l'arène publique où l'on est capable d'essuyer un outrage dans l'hic et nunc et de déplacer sa colère dans l'espace civique du tribunal ${ }^{55}$.

Mais on ajoutera que cette tendance n'est pas une innovation radicale : on a vu qu'Achille, outragé dans sa timè, rumine entre deux choix : tuer l'offenseur Agamemnon et laver la honte ou bien contrôler sa célèbre fureur meurtrière et le frapper avec la force de ses injures. L'intervention d'Athéna représente de façon paradigmatique, déjà chez Homère, la priorité de la maîtrise de soi et l'importance d'être, dans la louange comme dans l'injure, un « bon diseur de mots ${ }^{56}$.

\section{BIBLIOGRAPHIE}

Austin J. L. (1962), How to Do Things with Words, Oxford.

Benveniste É. (1969), Le vocabulaire des institutions indoeuropéennes, II, Paris.

Boehm C. (1987), Blood Revenge : The Anthropology of Feuding in Montenegro and Other Tribal Societies, Philadelphia.

Campbell J. K. (1964), Honour, Family and Patronage : A Study of Institutions and Moral Values in a Greek Mountain Community, Oxford.

Cantarella E. (2002), Itaca. Eroi, donne, potere tra vendetta e diritto, Milan.

Carlier P. (2003), « Regalità omeriche e regalità greche dell'alto arcaismo », Luppino Manes E. ed., Storiografia e regalità nel mondo greco, colloquio disciplinare (Chieti, 17-18 gennaio 2002), Chieti.

Daly M. et Wilson M. (1988), Homicide, New York.

Davis J. (1977), People of the Mediterranean. An Essay in Comparative Social Anthropology, Londres.

Donlan W. (1981), « Reciprocities in Homer », Classical World 75, p. 137-175.

Donlan W. (1982), « The Politics of Generosity in Homer », Helios 9, p. 1-15.

Eibl-Eibesfeldt I. (1979), The Biology of Peace and War, Londres. 
Finkelberg M. (1998), « Timè and Aretè in Homer », CQ 48, p. 15-28.

Finley M. I. (1982), The World of Odysseus, Cambridge.

Finley M. I. (1983), Politics in the Ancient World, Cambridge.

Fisher N. R. E. (1992), Hybris : A Study in the Values of Honour and Shame in Ancient Greece, Warminster.

Fiume G. éd. (1989), Onore e storia nelle società mediterranee, Palerme.

Gagné R. (2013), Ancestral Fault, Cambridge.

Gernet L. (2001), Recherches sur le développement de la pensée juridique et morale en Grèce, Paris.

Gilmore D. D. éd. (1987), Honor and Shame and the Unity of the Mediterranean, A Special Publication of the American Anthropological Association, n. 22, Washington D.C.

Giordano M. (1999), La parola efficace. Maledizioni, giuramenti e benedizioni in Grecia antica, PiseRome.

Giordano M. (2010), Iliade I, la peste - l'ira, introduzione e commento, Rome.

Herman G. (2006), Morality and Behaviour in Democratic Athens. A Social History, Cambridge.

Horden P. et Purcell N. (2000), The Corrupting Sea. A Study of Mediterranean History, Oxford.

Laslett P. (1956), « The Face to Face Society », dans Laslett P. éd., Philosophy, Politics and Society, Oxford, p. 157-184.

Lincoln B. (1994), Authority. Construction and Corrosion, Chicago-Londres.

MacHardy F. (2008), Revenge in Athenian Culture, Londres.

Maravall J. A. (1984), Poder, honor y élites en el siglo XVII, Madrid.

Martucci D. (2010), I Kanun delle montagne albanesi. Fonti, fondamenti e mutazioni del diritto tradizionale albanese, Bari.

Ober J. (1996), The Athenian Revolution. Essays on Ancient Greek Democracy and Political Theory, Princeton.

Osborne R. (1985), Demos : The Discovery of Classical Attika, Cambridge.

Peristiany J. G. éd. (1965), Honour and Shame. The Values of Mediterranean Society, Londres.

Peristiany J. G. et Pitt-Rivers J. éd. (1991), Honour and Grace in Mediterranean Society, Cambridge.

Pina-Cabral de J. (1989), « The Mediterranean as a Category of Regional Comparison : a Critical View », Current Anthropology 30, p. 399-406.

Rappaport R. (1984), Pigs for the Ancestors : Ritual in the Ecology of a New Guinea People, New Haven.

Resta P. (2002), Pensare il sangue. La vendetta nella cultura albanese, Rome.

Roisman J. (2005), The Rhetoric of Manhood. Masculinity in the Attic Orators, Berkeley.

Scheid-Tissinier É. (1994), Les usages du don chez Homère, Nancy.

Scheid-Tissinier É. (2005), Remarques sur les fondements de la vengeance en Grèce archaïque et classique , dans Bertrand J.-M. éd., La violence dans les mondes grec et romain, actes du colloque international (Paris, 2-4 mai 2002), Paris, p. 395-410. 
Scheid-Tissinier É. (2007), « Le rôle de la colère dans les tribunaux athéniens », dans Schmitt Pantel P. et Polignac de F. éd., Athènes et le politique. Dans le sillage de Claude Mossé, Paris, p. 179-198.

Schneider J. et Schneider P. (1976), Culture and Political Economy in Western Sicily, New York.

Stewart F. H. (1994), Honor, Chicago-Londres.

Svenbro J. (1980), «Vengeance et société en Grèce archaïque. À propos de la fin de l'Odyssée », dans Verdier R. et Poly J.-P. éd., La vengeance. Vengeance, pouvoirs et idéologies dans quelques civilisations de l'Antiquité, Paris, p. 47-63.

Valentini G. éd. (1968), La legge delle montagne albanesi nella relazione della Missione Volante, Florence.

Verdier R. (1980), « Le système vindicatoire », dans Verdier R. éd., La vengeance. La vengeance dans les sociétés extra-occidentales, vol. 1, Paris, p. 13-41.

\section{NOTES}

1. Giordano 1999, p. 50-51.

2. La plupart des malédictions sont prononcées in absentia, par exemple Iliade, II, 417-418; Odyssée , XVII, 251-253 ; XVIII, 235-242. Voir aussi, de façon générale, l'utilisation des malédictions conditionnelles dans la loi ou dans les serments. Dans le même sens, on remarquera que le destinataire de la malédiction n'est souvent pas le destinataire de l'énonciation au point de vue linguistique. Voir sur ce sujet Giordano 1999, p. 19-25.

3. Sur les dynamiques de la faute ancestrale, voir l'excellent travail de Renaud Gagné 2013.

4. Cantarella 2002, p. 33.

5. Peristiany 1965 ; voir aussi Peristiany 1991.

6. Dans la même lignée que Peristiany, Gilmore 1987 ; contra, Pina-Cabral 1989; pour une évaluation récente voir Horden et Purcell 2000, p. 485 sq.

7. Davis 1977 ; Fiume 1989.

8. Voir en particulier Schneider et Schneider 1976.

9. Sur les dons, voir Scheid-Tissinier 1994. Sur le rapport entre objets et autorité voir Lincoln 1994, p. 7 sq. La culture homérique est une culture d'objets, dans le sens que presque tout passe à travers les objets ; la cause de la guerre de Troie est l'enlèvement d'une femme, possession et part d'honneur de son mari, tout comme la rage d'Achille est déclenchée par la privation de son geras - en l'occurrence, la captive Briséis. Sur ces aspects, voir Giordano 2010, p. 38-46 et passim. Voir Finkelberg 1998, p. 16, pour la formule épique emmore times et l'idée de "allotment » of timé répandue dans le patrimoine épique.

10. Cantarella 2002, p. 33.

11. Voir, par exemple, le récit de l'Éthiopide (éd. Davis, 1988, p. 47, 1. 7-13), dans lequel Achille tue Thersite pour répondre aux injures et aux reproches dont son amour pour Penthésilée avait été l'objet; dans Sophocle, @Edipe roi, 800-812, ๔Edipe raconte comment il a tué Laios et ses servants pour avoir été injurié. Sur ce passage, voir MacHardy 2008, p. 100.

12. Cantarella 2002, p. 26.

13. Verdier 1980. Sur honneur, insulte et vengeance, voir MacHardy 2008, p. 85-86.

14. Austin 1962. Sur la notion de "face-to-face society » Laslett 1975, qui a été appliquée au cas grec par Finley 1983, p. 28 sq. et passim; l'application de cette définition à la Grèce antique a été réfutée par Osborne 1985, p. 65 et Ober 1996. 
15. Cette sélection, je l'avoue, est hautement restrictive et sert la finalité de mon exposé : le code de l'honneur, comme je le décris ici, régit un grand nombre de sociétés étudiées par les anthropologues sur une échelle diachronique très vaste.

16. Campbell 1964, p. 268.

17. Campbell 1964, p. 269.

18. Bien sûr, cela ne concerne pas exclusivement les Sarakatsani. Pour l'Athènes antique, voir les remarques de Roisman 2005, p. 14-15. L'auteur souligne comment l'extrême sensibilité des jeunes hommes face à l'honneur était vue de façon négative dans la mesure où cette sensibilité «does not always sit well with the values of moderation and self-control », valeurs prioritaires de la société athénienne.

19. Campbell 1964, p. 164.

20. Ce Kanun est attribué au personnage de Lek Dukagjini, un prince ayant vécu entre 1410 et 1479 , et qui n'est assurément pas l'auteur de ce code ; voir Martucci 2010, p. 24-30.

21. Sur les autres Kanun dans le sud du pays, Martucci 2010, p. 13-16.

22. Pour les sentences, voir Martucci 2010, p. VIII, n. 5.

23. $§ 596$.

24. Resta 2002, p. 129.

25. Ne pas se venger est une honte inadmissible pour la société albanaise : "Chi non è ancora riuscito a prendere il sangue del suo nemico, non è onorato tra i suoi. Nelle adunanze che spesso si fanno tra i montanari per feste o inviti, l'arma di tal uomo non può stare con quelle degli altri, ma dev'essere posta in disparte. Passerà la rakia od acquavite, ed a lui solo non si riempirà il bicchierino, perché non ha ancora preso il suo sangue. » (Valentini 1968, p. 5).

26. Resta 2002, p. 124. P. Resta (2002, p. 102-121) relate un cas de vengeance dans le village de Lapardha, dans la zone méridionale de la Labëria, au début de notre siècle, déclenché par des injures prononcées pendant une querelle sur les frontières de pâturage.

27. Resta 2002, p. 126.

28. Martucci 2010, p. 179.

29. La connexion entre ivresse et la chaîne insulte-violence jusqu'à l'homicide est physiologique et donc « universelle » : voir par exemple pour les États-Unis Daly et Wilson (1988, p. 124-125).

30. Parmi lesquelles je me permets de renvoyer à Giordano 2010.

31. Souvent la querelle est résumée à une réponse d'Achille à la soustraction de Briséis ; ainsi par exemple MacHardy (2008, p. 87-88 sq.) lie la querelle à la « reproductive competition ». Comme je vais le souligner, la querelle part du défi d'Achille et se déroule autour des tentatives réciproques d'ébranler la timè des parties prenantes.

32. Voir dans ce sens Lincoln 1994, p. 14-18 sq.

33. Les traductions de l'Iliade sont de Mazon, avec quelques modifications.

34. Voir Stewart 1994, p. 64.

35. Voir sur ce point Giordano 2010, p. 25-30.

36. Voir MacHardy 2008, p. 94-95.

37. Rappaport 1984, p. 106 sq.

38. Eibl-Eibesfeldt 1979, p. 102-110.

39. Vers 225- 233: «Sac à vin! CEil de chien et cœur de cerf! Jamais tu n'as eu le courage de t'armer pour la guerre avec tes gens, ni de partir pour un aguets avec l'élite achéenne : tout cela te semble la mort! Certes il est plus avantageux, sans s'éloigner du vaste camp des Achéens, d'arracher les présents qu'il a reçus à quiconque te parle en face. Ah ! Le beau roi, dévoreur de son peuple! Il faut qu'il commande à des gens de rien sans quoi, fils d'Atrée, tu aurais aujourd'hui lancé ton dernier outrage ".

40. W. Donlan (1982 et 1981) le voit en concurrence avec un système fixe. Sur les limites de ce modèle voir Carlier 2003.

41. Benveniste 1969, p. 75. 
42. J'emprunte cette définition à F. H. Stewart (1994, p. 54-63).

43. Voir Finkelberg 1998, p. 15 sq., sur l'importance de l'émulation mutuelle entre pairs pour la définition de arete. Dans le monde homérique, on s'injurie entre rois ou entre pairs, tandis qu'un inférieur n'a pas le droit d'adresser des reproches aux rois. Dans un épisode paradigmatique du chant II de l'Iliade, Thersite insulte Agamemnon avec des raisons semblables à celles d'Achille, mais il parle hors-mesure (v. 213- 214, akosma, ou kata kosmon). En tant qu'inférieur, il est battu et réprimandé par Ulysse, Iliade, II, 246-266. L'épisode de Thersite est d'un intérêt particulier pour notre sujet et mériterait une analyse plus détaillée.

44. Finley 1982, p. 112. Voir aussi Odyssée, X, 428-448, où Euryloque insulte Ulysse en l'appelant « lâche ». Ulysse est sur le point de le tuer, mais ses compagnons le dissuadent en l'implorant. Voir aussi Odyssée, XVII, 453-487 ; Ulysse déguisé en mendiant est maltraité publiquement par Antinoos, il répond aux insultes verbales et physiques par des malédictions, mais évite la violence pour ne pas se dévoiler comme un homme de rang. Il ne tardera pas toutefois à se venger, une fois réinstauré dans son identité royale, au chant XXII.

45. Maravall 1984, p. 48, à propos du sens de l'honneur dans l'Espagne des XVI ${ }^{\mathrm{e}}$ et XVII ${ }^{\mathrm{e}}$ siècles.

46. Svenbro 1980, p. 55.

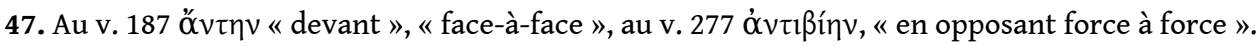

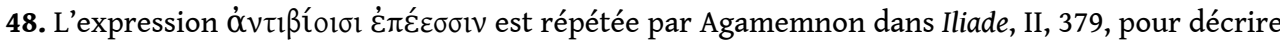
la querelle, tout en admettant cette fois sa responsabilité.

49. Le meurtre de Thersite par Achille, raconté dans l'Éthiopide ainsi que la stasis qui suit, montre ce qui peut arriver si l'on ne se borne pas aux mots.

50. MacHardy 2008, p. 98 ; cf. par exemple Platon, Gorgias, 486 a-c.

51. Un passage du discours contre Conon de Démosthène (LIV, 17-19) éclaircit précisément ce point. Sur le contrôle des réponses par la vengeance dans les conflits, voir notamment Herman 2006, p. 155-206.

52. Scheid-Tissinier 2005, p. 395-410.

53. Sur le plan des réponses législatives, cette parité à Athènes est assurée pour tous les citoyens considérés justement comme des pairs, mais excluait une large portion de la population (femmes, esclaves, étrangers résidents). Il reste aussi bien entendu que le pouvoir personnel, en termes politiques et économiques, avait un poids énorme dans la possibilité de l'emporter au cours d'un procès, mais le fait reste que le système athénien donnait une possibilité égale à chaque citoyen d'avoir recours au tribunal pour se défendre.

54. Démosthène, Contre Midias, XXI, 71-76, invoque la mort de son offenseur, et cite le cas d'Euaion qui avait tué un certain Boiôtos qui l'avait frappé, par un acte de hybris; le meurtrier dit que « ce ne fut pas le coup en soi [qui provoqua] la fureur homicide mais le déshonneur (atimia)», XXI, 72 ; Aristote, Rhétorique, 1378b 29 sq., sur la connexion entre hybris, atimia et orgè, voir Scheid-Tissinier 2007, en particulier p. 182-184.

55. Sur ces dynamiques voir la monographie de Herman 2006.

56. On ne peut entrer ici dans le débat sur l'existence d'une continuité ou d'une rupture entre la culture homérique et la culture athénienne sur l'utilisation de la violence et, donc de la vengeance. Voir Herman 2006, passim, et contra MacHardy 2008, p. 97- 99. 


\section{RÉSUMÉS}

Cet article explore la relation que l'injure entretient avec l'honneur et la vengeance, à l'aide de quelques données comparatives (Sarakatsani, Albanie, Nouvelle Guinée), pour montrer comment injure, honneur et vengeance forment un véritable système. J'aborde de ce point de vue la querelle entre Achille et Agamemnon au premier chant de l'Iliade pour montrer - à la lumière du modèle anthropologique du combat rituel - que l'honneur disputé par le moyen de l'injure est agonal en soi. L'injure à son tour a pour fonction de mettre à l'épreuve l'honneur et les règles sociales qui lui sont liées et elle fonctionne en même temps comme une mise à l'épreuve permanente de la valeur et de l'identité. L'injure apparaît dès lors comme un élément et une pratique intégrés étroitement au fonctionnement de la société homérique. Le guerrier homérique doit savoir manier les outils d'une rhétorique polémique, non seulement pour faire valoir sa parole à l'assemblée et au conseil, convaincre les autres et vaincre les adversaires, mais il lui faut aussi une parole qui sache blesser sans dommage son pair dans l'arène interne des égaux. L'article propose d'étendre ces considérations à Athènes, de manière à ébaucher l'évolution de ce système dans la diachronie, en montrant comment le guerrier homérique tout comme le citoyen athénien doit savoir mettre à l'épreuve l'honneur des autres par la parole, tout en évitant le basculement dans la violence physique.

This paper explores the way insult, honour, and revenge relate to each other, by the aid of comparative cases (Sarakatzani, Albania, New Guinea), to show that insult, honour, and revenge form a veritable system. Approaching the dispute of Achilles and Agamemnon in the first book of the Iliad, also on the account of the anthropological model of ritual combats, it is further argued that the honour disputed by means of insulting the rival is agonistic. Insult in its turn upholds the function of proving honour as well as the social rules thereby connected, including individual value and identity. The social model of insult resorting from this analysis is conducive to considering it an integral practice of the Homeric world, insofar as the Homeric warrior 'doer of deeds and speaker of words' should master a 'battle rhetoric' not only in order to persuade and win others' consent in the assembly, but also to challenge without physical damage his equal by mastering the tool of insulting words. The paper ends in Athens, where a diachronic perspective is outlined, highlighting how both the Homeric warrior and the Athenian citizen are invited to prove other equals' honour mastering their aggressive impulse by using insult, and avoiding murderous revenge and physical violence.

\section{INDEX}

Keywords : offense, honour, revenge, Homer, Greek law, ritual combat

Mots-clés : injure, honneur, vengeance, Homère, droit grec, combat rituel 


\section{AUTEUR}

MANUELA GIORDANO

Université de Calabre 